\title{
Rendimiento académico y la calidad del desayuno en estudiantes de la Facultad de Bromatología y Nutrición Universidad Nacional José Faustino Sánchez Carrión Huacho 2015
}

Academic Performance and Quality breakfast is students of the Faculty of Food Science and Nutrition at the National University Jose Faustino Sanchez Carrión Huacho 2015 Laura Montes Carrasco ${ }^{1}$, Eufemio Macedo Barrera', Mirtha Sussan Trejo López ${ }^{2}$

\section{RESUMEN}

Objetivo: Conocer la relación entre el rendimiento académico y calidad del desayuno en estudiantes de la facultad de Bromatología y Nutrición. Material y Métodos: Muestra 244 (18 a 25 años), distribuidos 195 mujeres y 49 varones. Se aplicó encuesta frecuencia consumo. El rendimiento académico se midió la calificación media obtenida al término de los cursos obligatorios y categorizados en consideración al reglamento académico. Resultados: La mayoría pertenece al género femenino $79,92 \%$, y la masculina $20,08 \%$. La calidad del desayuno de la población en estudio se observó que, una minoría toma un desayuno completo, $79 \%$. El consumo de un desayuno $\%$, no de buena calidad es de $18,03 \%$, de mejorable calidad representa $29,92 \%$ insuficiente calidad $31,15 \%$ y de mala calidad $13,11 \%$. La calidad del desayuno y rendimiento académico, se ha agrupado en tres categorías; y sirvió para la contratación de la hipótesis, los estudiantes que consumen desayuno de mala calidad representan una calificación de rendimiento académico de deficiente $11,1 \%$, aceptable $31,1 \%$ y bueno con $1,6 \%$. Los estudiantes que consumían desayuno de buena calidad están calificados en el rendimiento académico como deficiente $11,1 \%$, aceptable $34,8 \%$ y bueno $11,9 \%$.Los estudiantes que consumen desayuno completo están calificados con respecto a la variable rendimiento académico con aceptable $4,5 \%$ y bueno 2,9\%. Conclusión: El 0,54\% consumen un desayuno insuficiente, y el $13,11 \%$ no toma desayuno. Es recomendable que los estudiantes tomen un desayuno equilibrado para demandas intelectuales de la educación. Se recomienda comer variado, los estudiantes que varían el desayuno tendrán una dieta más sana, en comparación con los que no lo hacen. Cuando se relacionó la calidad del desayuno y el rendimiento académico se estableció que conforme mejora

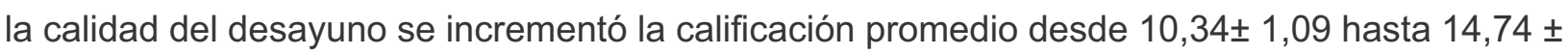
1,28 para los estudiantes con desayuno completo.

Palabras clave: Desayuno, rendimiento académico.

\section{ABSTRACT}

Objective: to know the relation between the academic performance and quality of breakfast in students of the faculty of Bromatology and Nutrition. Material and Methods: Sample 244 (18 to 25 years old), distributed 195 women and 49 men. Frequency consumption survey was applied. Academic performance was measured by the average grade obtained at the end of the compulsory courses and categorized in consideration of the academic regulations. Results: The majority belongs to the feminine gender $79.92 \%$ and the masculine $20,08 \%$. The breakfast quality of the study populations was observed that, a minority take a full breakfast, $79 \%$. The consumption of a

\footnotetext{
1 Facultad de Bromatología y Nutrición. Universidad Nacional José Faustino Sánchez Carrión. Huacho, Perú.

${ }^{2}$ Facultad de Ciencias. Universidad Nacional José Faustino Sánchez Carrión, Huacho, Perú.
} 
breakfast not of good quality is $18.03 \%$, of improvable quality represents $29.92 \%$ insufficient quality $31.15 \%$ and poor quality $13.11 \%$. The quality of breakfast and academic performance, has been grouped into three categories; and served to test the hypothesis, students who consume poor quality breakfast represent an academic performance rating of deficient $11.1 \%$, acceptable $31.1 \%$ and good with $1.6 \%$. Students who consumed good quality breakfast are classified in academic performance as deficient $11.1 \%$, acceptable $34.8 \%$ and good $11.9 \%$. Students who consume full breakfast are rated with respect to the variable academic performance as acceptable $4.5 \%$ and good $2.9 \%$. Conclusion: $0.54 \%$ consume an insufficient breakfast, and $13.11 \%$ do not eat breakfast. It is recommended to eat varied; students who vary breakfast will have a healthier diet, compared to those who do not. When breakfast and academic performance were related, it was established that as the quality of breakfast improved the average score increased from 10.34

\section{INTRODUCCIÓN}

El desayuno es la primera comida importante $y$, de alguna manera, condiciona la ingesta de alimentos y de nutrientes en el equilibrio nutricional diario. Diversos estudios ponen de manifiesto desde hace tiempo la influencia que el desayuno tiene sobre el rendimiento físico e intelectual en las actividades realizadas durante la mañana (Herrero, 2008). La contribución del desayuno a la ingesta energética sugiere que debería aportar entre un 25 y $30 \%$ de las necesidades diarias, y su calidad nutricional se favorecería por la inclusión de lácteos, frutas y cereales (Nicklas, 1998). Es habitual tomar un primer desayuno de poca calidad nutricional y se complementa a media mañana con alimentos clasificados como no saludables, sobre todo, cuando la jornada escolar es de mañana exclusivamente (Nuñez, 1998).

La omisión o la ingesta de un desayuno insuficiente o deficiente, puede repercutir en las actividades físicas e intelectuales de los adolescentes como disminución de la atención y del rendimiento académico, especialmente en las primeras horas de clase. La influencia de la calidad del desayuno o de su omisión pueden influir en el rendimiento cognitivo y académico ya que parece que la función cerebral es sensible a variacio bien nutridos, el trabajo memorístico es sensible a la omisión del desayuno. Por el contrario, un buen desayuno mejora el rendimiento escolar y reduce el absentismo escolar, mejora el comportamiento y el control emocional, disminuye la ansiedad, hiperactividad, depresión o disfunciones psicosociales (Fernández et al., 2008).

El presente estudio tuvo como objetivo relacionar el rendimiento académico y calidad del desayuno en estudiantes de la Facultad de Bromatología y Nutrición.

\section{MATERIAL Y MÉTODOS}

El estudio se realizó en una muestra de 244 alumnos, obtenida mediante método aleatorio al azar, (49 varones y 195 mujeres) con edades comprendidas de 18 a 25 años, estudiantes del primer al décimo ciclo de la Facultad de Bromatología y Nutrición, a los que se les aplicó una encuesta que evaluó la frecuencia del consumo del desayuno. El rendimiento académico de los estudiantes encuestados fue proporcionado por la Oficina de Registros Académicos y en su evaluación se utilizó la calificación media obtenida al término de los cursos obligatorios representativos elegidos por el estudiante y categorizado tomando en consideración el Reglamento Académico General de la Universidad Nacional José Faustino Sánchez Carrión.

Para calificar los desayunos de los estudiantes se utilizó el siguiente criterio:

Desayuno completo. Aporta $25 \%$ de las necesidades diarias de energía e incluye 
alimentos de al menos cuatro grupos distintos lácteos, cereales, frutas, aceites y grasas.

Buena Calidad: contiene un alimento, al menos del grupo de lácteos, cereales y fruta. Mejorable calidad: falta uno de los grupos. Insuficiente calidad: faltan dos de los grupos. Mala Calidad: No desayuna.

Los datos recogidos fueron procesados mediante el programa SPSS 21.0, mediante la estadística descriptiva e inferencial. Para la contrastación de la hipótesis se utilizó la estadística no paramétrica empleando el coeficiente de correlación Tau-B de Kendall apropiado para variables ordinales.

\section{RESULTADOS}

De la muestra estudiada se encontró que la mayoría pertenece al género femenino representado el 79,92 \%; mientras que la masculina, $20,08 \%$ tal como se muestra en la Figura 1.

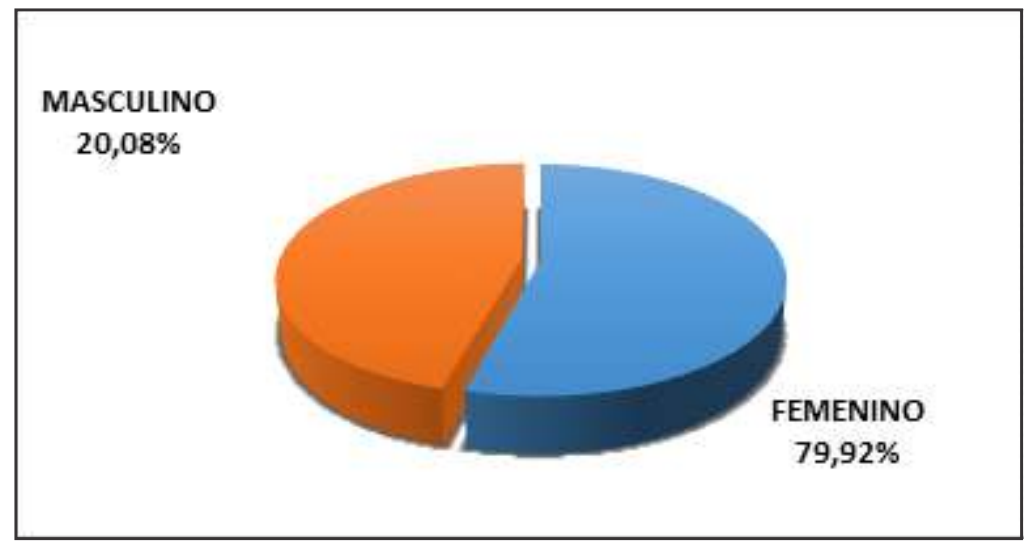

Figura 1. Distribución de la muestra encuestada según género

Tabla 1. Clasificación del consumo de desayuno en estudiantes

\begin{tabular}{lcc}
\hline \multicolumn{1}{c}{ Calidad del Desayuno } & $\begin{array}{c}\mathbf{N}^{\circ} \\
\text { Estudiantes }\end{array}$ & Porcentaje \\
\hline Desayuno completo & 19 & 7,79 \\
Buena calidad & 44 & 18,03 \\
Mejorable calidad & 73 & 29,92 \\
Insuficiente calidad & 76 & 31,15 \\
Mala calidad & 32 & 13,11 \\
\hline \multicolumn{1}{c}{ Total } & $\mathbf{2 4 4}$ & $\mathbf{1 0 0 , 0 0}$ \\
\hline
\end{tabular}

En la Tabla 1 y Figura 2 se observa que, una minoría de la muestra toma un desayuno completo, $7,79 \%$. En tanto que desayuno de buena calidad alcanza a $18,03 \%$ de la muestra, de mejorable calidad representa $29,92 \%$, insuficiente calidad $31,15 \%$ y de mala calidad $13,11 \%$. 


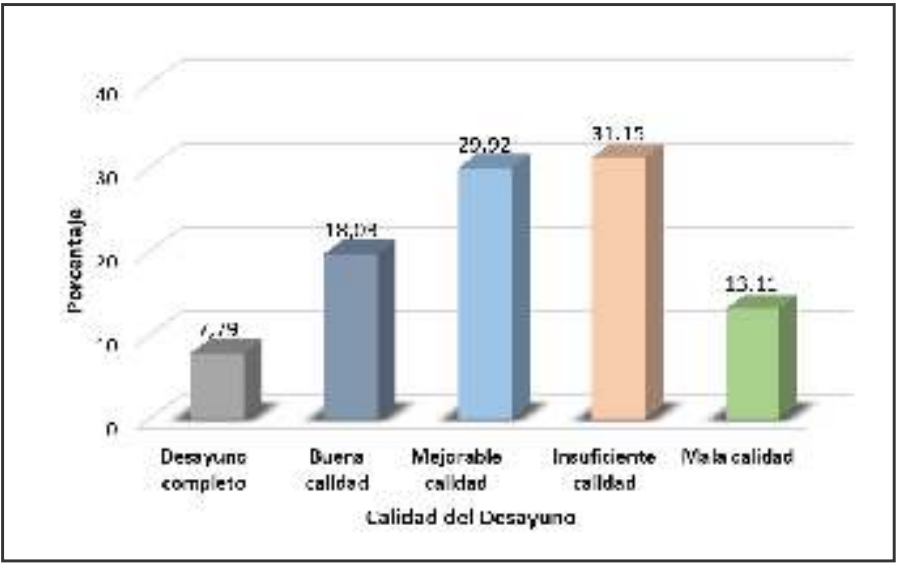

Figura 2. Clasificación del consumo de desayuno en estudiantes

Los alimentos que no se incluyó en su mayoría son frutas, leche, yogur entre otros.

Tabla 2. Clasificación del consumo de desayuno en estudiantes según género

\begin{tabular}{lcccccc} 
& \multirow{2}{*}{ DESAYUNO } & $\mathbf{N}^{\circ}$ & \multicolumn{5}{c}{ Genero } \\
\cline { 3 - 7 } & Estudiantes & Femenino & $\%$ & Masculino & $\%$ \\
\hline Desayuno completo & 19 & 15 & 6,15 & 4 & 1,64 \\
\hline Buena calidad & 44 & 37 & 15,16 & 7 & 2,87 \\
\hline Mejorable calidad & 73 & 56 & 22,95 & 17 & 6,96 \\
\hline Insuficiente calidad & 76 & 60 & 24,59 & 16 & 6.56 \\
\hline Mala calidad & 32 & 27 & 11,07 & 5 & 2,05 \\
\hline Total & 244 & 195 & 79,92 & 49 & 20,08 \\
\hline
\end{tabular}

La Tabla 2, indica que la minoría de estudiantes $6,15 \%$, del género femenina, consume un desayuno completa. Mientras que de buena calidad solo $15,16 \%$, así mismo de calidad insuficiente un $24,59 \%$ y de mala calidad $11,07 \%$. Con respecto al género masculino, la minoría de estudiantes 1,64\%, toma un desayuno de calidad completa, de buena calidad solo el 2,87 \%, de mejorable calidad $6,96 \%$, y de mala calidad $2,05 \%$ de estudiantes.

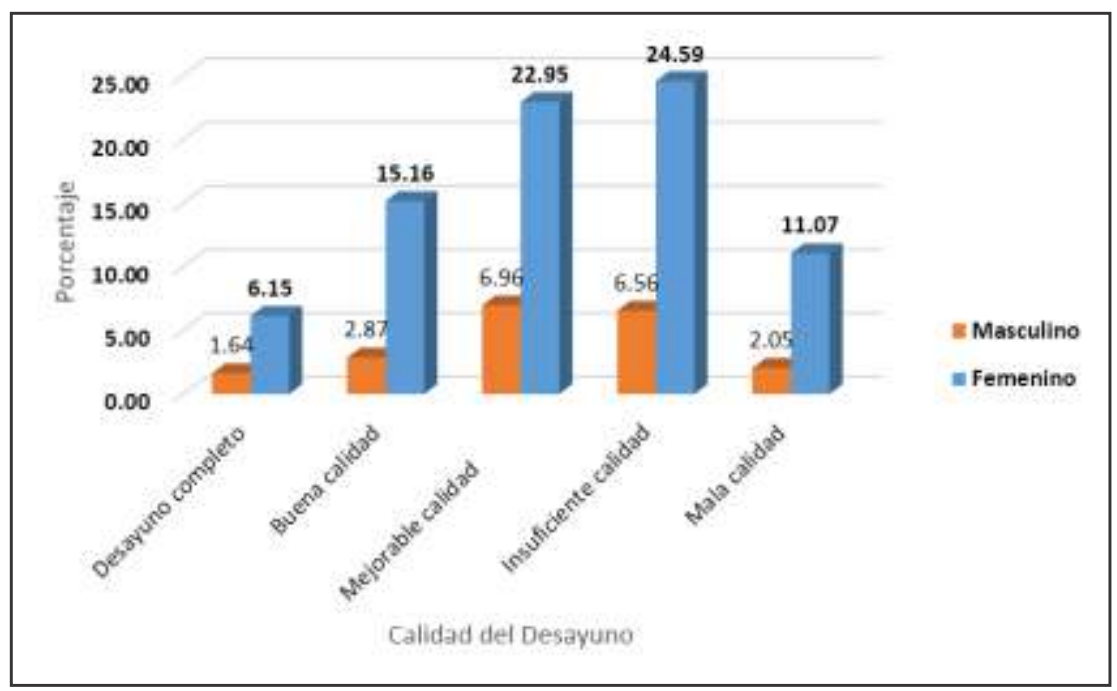

Figura 3. Clasificación del consumo de desayuno en estudiantes según género 
En la Figura 3, se observa el comportamiento de la población femenina y masculina notándose que es mayor la cantidad de estudiantes mujeres que no desayunan, a diferencia de los estudiantes varones. Así mismo, consumen un desayuno deficiente en nutrientes y energía.

Tabla 3. Número y porcentaje de la clasificación de consumo desayuno en estudiantes

\begin{tabular}{lcccc}
\hline \multirow{2}{*}{ DESAYUNO } & $\begin{array}{c}\text { No } \\
\text { Estudiantes }\end{array}$ & $\begin{array}{c}\text { Porcentaje } \\
\text { \% }\end{array}$ & \multicolumn{2}{c}{ Calificación } \\
\hline Desayuno completo & 19 & 7,78 & Media & DS \\
\hline Buena calidad & 44 & 18,02 & 14746 & 0,822 \\
\hline Mejorable calidad & 73 & 29,91 & 13,197 & 1,126 \\
\hline Insuficiente calidad & 76 & 31,14 & 12,665 & 1,293 \\
\hline Mala calidad & 32 & 13,11 & 11,400 & 1,063 \\
\hline \multicolumn{1}{c}{ Total } & $\mathbf{2 4 4}$ & $\mathbf{1 0 0}$ & 10,431 & 1,319 \\
\hline \multicolumn{1}{c}{} & & & \\
\hline
\end{tabular}

En la Tabla 3, da conocer que el consumo de un desayuno completo corresponde al $7,78 \%$ del total de la muestra, con un promedio alto $14,74 \pm 0,82$. Los valores intermedios se encontraron para el desayuno de mejorable calidad e insuficiente calidad con $29,91 \%$ y $31,14 \%$ respectivamente. Mientras que el $13,11 \%$ tuvo el promedio más bajo 10,43 $\pm 1,319$.

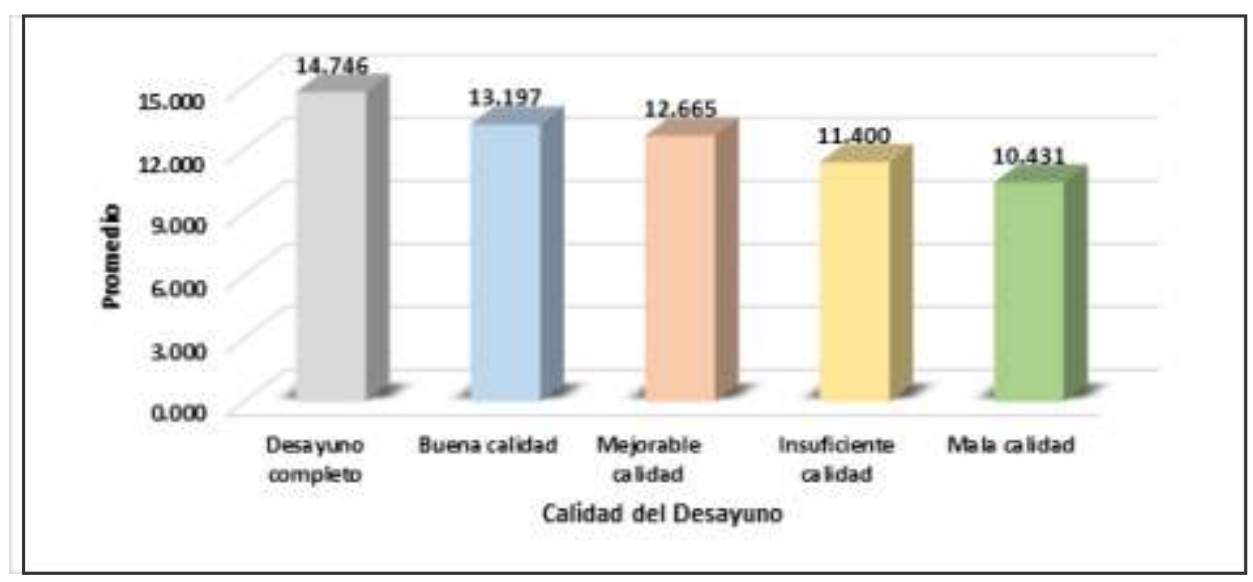

Figura 4. Número y porcentaje de la clasificación de consumo de desayuno según calificación

En la Figura 4, se aprecia el cambio significativo del desplazamiento de las calificaciones promedio, la media y desviación de las calificaciones obtenidas en la calidad del desayuno, se observa que conforme mejora la calidad del desayuno este se incrementa desde $10,43 \pm 1,31$ para el grupo de desayuno de mala calidad, hasta un valor medio de 14,74 $\pm 0,82$ que corresponde al grupo de calidad completa. 
Tabla 4. Distribución de estudiantes según calidad del desayuno y rendimiento académico

\begin{tabular}{|c|c|c|c|c|c|c|c|c|}
\hline \multirow{3}{*}{$\begin{array}{c}\text { DEL } \\
\text { DESAYUNO }\end{array}$} & \multicolumn{6}{|c|}{ RENDIMIENTO ACADÉMICO } & \multirow{2}{*}{\multicolumn{2}{|c|}{ TOTAL }} \\
\hline & \multicolumn{2}{|c|}{ DEFICIENTE } & \multicolumn{2}{|c|}{ ACEPTABLE } & \multicolumn{2}{|c|}{ BUENO } & & \\
\hline & $\mathbf{N}^{\circ}$ & $\%$ & $\mathbf{N}^{\circ}$ & $\%$ & $\mathbf{N}^{0}$ & $\%$ & $\mathbf{N}^{\circ}$ & $\%$ \\
\hline Mala calidad & 27 & 11,1 & 76 & 31,1 & 4 & 1,6 & 107 & 43,9 \\
\hline $\begin{array}{l}\text { Buena } \\
\text { calidad }\end{array}$ & 5 & 2,0 & 85 & 34,8 & 29 & 11,9 & 119 & 48,8 \\
\hline $\begin{array}{l}\text { Desayuno } \\
\text { completo }\end{array}$ & 0 & 0,0 & 11 & 4,5 & 7 & 2,9 & 18 & 7,4 \\
\hline TOTAL & 32 & 13,1 & 172 & 70,5 & 40 & 16,4 & 244 & 100,0 \\
\hline
\end{tabular}

La Tabla 4 muestra, el rendimiento académico, en relación a la calidad del desayuno, indicándose que las categorías mejorable calidad e insuficiente calidad de acuerdo a los resultados fueron distribuidos, según el rendimiento académico, en los niveles deficiente, aceptable y bueno.

Los estudiantes que cosumían un desayuno de mala calidad fueron calificados con un rendimiento académico de deficiente $11,1 \%$, aceptable $31,1 \%$ y bueno con $1,6 \%$. Los estudiantes que consumían desayuno de buena calidad se clasificaron con un rendimiento académico como deficiente $11,1 \%$, aceptable $34,8 \%$ y bueno $11,9 \%$. Los estudiantes que consumían desayuno completo están calificados con respecto a la variable rendimiento académico con aceptable $4,5 \%$ y bueno $2,9 \%$.

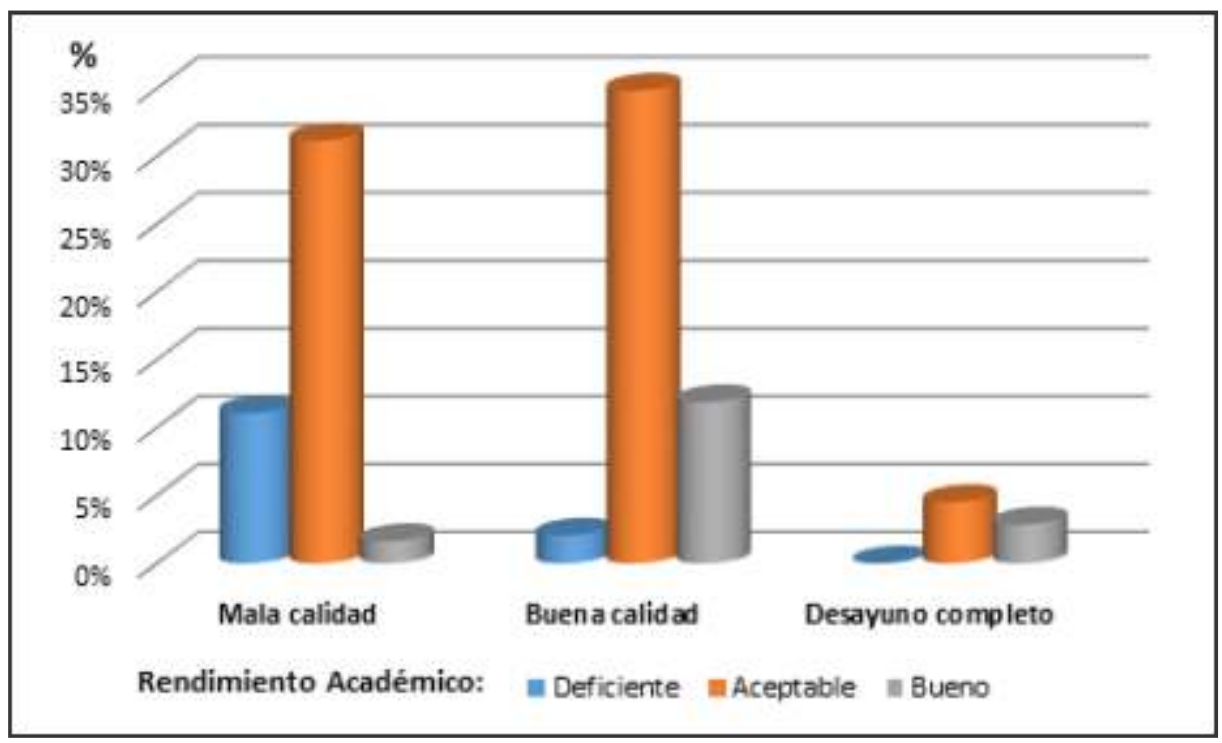

Figura 5. Distribución porcentual de los estudiantes por calidad de desayuno y rendimiento académico

En la Figura 5, se aprecia que en las diversas calidades de desayuno que consume la población estudiantil alcanza un aceptable rendimiento académico, pero se acentúa con un alto predominio cuando el desayuno es de buena calidad. 
Tabla 5. Análisis de la Variables utilizando la Prueba Tau-b de Kendall

\begin{tabular}{|c|c|c|c|}
\hline Prueba & Valor & $\begin{array}{c}\text { Error } \\
\text { Estándar }\end{array}$ & Significancia \\
\hline Valor Tau -b de Kendall & 0,388 & 0,044 & 0,000 \\
\hline
\end{tabular}

En la Tabla 5, observamos que la significancia 0,000 es menor que el nivel de significación 0,05 , por lo tanto, se rechaza $\mathrm{H}_{\mathrm{o}}$ y se concluye que la calidad del desayuno está influyendo directamente en el rendimiento académico de los alumnos de Bromatología y Nutrición de la Universidad Nacional José Faustino Sánchez Carrión.

\section{DISCUSIÓN}

Diversos estudios indican el papel que desempeña el desayuno en el rendimiento académico de los estudiantes de diferentes edades y nivel socioeconómico. Así mismo refieren que, la omisión del desayuno provocaba una disminución del rendimiento físico en el transcurso de la mañana (Bayano y López, 2014).

El beneficio del desayuno sobre el rendimiento intelectual y equilibrio nutricional interviene en todas las etapas del aprendizaje, también en la edad adulta y en la edad avanzada. Mejorando la primera comida del día y tendrá, probablemente repercusiones positivas en el estado nutritivo y de salud del sujeto (Serra, 2000). La mayoría de la población en estudio está conformada por jóvenes adultas del género femenino. Esto puede deberse a que en su mayor parte la población de estudiantes de la Facultad de Bromatología y Nutrición está constituido por mujeres.

De acuerdo a la calidad conjunta del desayuno que consumen los estudiantes, se aprecia que la mayoría de la población $61,54 \%$ consume un desayuno insuficiente, deficiente en nutrientes, y el $13,11 \%$ no toma desayuno. Estudios realizados indican que es recomendable que los estudiantes tomen un desayuno equilibrado para enfrentarse a las demandas intelectuales de la educación (Pérez 1998). Los especialistas recomiendan comer un poco de todo y variado, con lo cual podría decirse que los estudiantes que varían el desayuno tendrán una dieta más sana en comparación con aquellos que no lo hacen (Farré, et al., 1998). Así mismo, López et al., (1997) comprobó que la omisión del desayuno tenía unos efectos adversos: en el estado emocional de los niños; en el rendimiento del trabajo físico-muscular.

O'Neil y Berenson (1998), refieren que la ingesta de lácteos, cereales y fruta en el desayuno contribuye a complementar el aporte adecuado de energía, carbohidratos, proteínas, lípidos, vitaminas y minerales a lo largo del día, un déficit en el consumo de nutrientes ocasionaría debilidad, cansancio, baja atención u otros signos y síntomas que se relacionan con el rendimiento, en la etapa escolar se da el máximo desarrollo intelectual, por el inicio de los pensamientos concretos y abstractos.

La gran minoría de población de estudiantes hombres y mujeres ingieren un desayuno de calidad completa con un aporte de energía de promedio de $655 \mathrm{Kcal}$, equivalente al $25 \%$ lo cual está de acuerdo a las recomendaciones establecidos por $2620 \mathrm{Kcal}$, de acuerdo al requerimiento de energía de la población peruana dado por el Ministerio de Salud Perú (2012). Los estudiantes que varían algo en su desayuno, tendrán una mejor calidad, dado que, en el desayuno se incluye de cuatro a cinco grupos alimenticios. Se ha podido establecer que la mayoría de los alumnos, no ingieren las calorías recomendadas para el desayuno, aunque algunos de ellos ingieren más o menos de esa cantidad (Pérez et al., 1998). Sánchez y Serra (2008) afirman que una ingesta calórica adecuada en el desayuno 
ejerce un efecto beneficioso en la memoria de forma inmediata, aunque no sobre la capacidad de concentración de los alumnos, lo cual reafirma que es más importante la calidad de lo que se come que la cantidad.

Cuando se observa la calidad del desayuno que toma la población de ambos géneros, se establece que la mayoría de la población femenina, consume un desayuno insuficiente en calidad debido a que no cumple con los aportes nutricionales requeridos, así mismo, es mayor la cantidad de estudiantes mujeres que no desayunan, a diferencia del masculino, lo que significa que ellos tienen mejores costumbres a pesar encontrarse en minoría, sin embargo los valores hallados para la población femenina puede deberse a que por ser personas adultas, tienen más libertad a la hora de tomar decisiones, con respecto al desayuno. El aporte en energía indica que existen diferencias entre ambos géneros, debido a la calidad y población femenina, La calidad del desayuno está directamente relacionada con la calificación media obtenida.

Los resultados encontrados son similares a los hallados por Fernández y Mateos (2008), indican que las chicas de 15-17 años son las que toman un desayuno de inferior calidad $y$ un $8,33 \%$ de estas alumnas omiten el desayuno, mientras que un $68,29 \%$ ingieren desayunos de calidad mejorable. Esta calidad del desayuno está directamente relacionada con la o menores calificación media obtenida. La población estudiada consume un desayuno poco adecuado lo que puede afectar a sus resultados académicos, especialmente de algunas asignaturas. Cuando se relaciona la calidad del desayuno y el rendimiento académico se establece que conforme mejora la calidad del desayuno se incrementa la calificación promedio desde $10.34 \pm 1.09$ hasta $14.74 \pm 1.28$ para los estudiantes que toman un desayuno completo.

Lo que está de acuerdo a lo indicado por otros estudios, que señalan que el consumo de un desayuno completo produce una mejora en la función cognitiva, comportamiento, atención, y participación, de los estudiantes. Asimismo, el estudio realizado coincide con los resultados hallados por Herrero y Fillat (2008), quienes señalan que conforme avanza la calidad del desayuno cambia su calificación significativamente la calificación promedio, hacia valores más elevados

Chávez et al. (1998) encontraron valores algo menores a los hallados en el estudio, sin embargo, establecieron que a medida que aumenta la calidad del desayuno, aumenta la calificación media desde un $6,18 \pm 1,89$ hasta $7,17 \pm 1,74$ en el grupo de alumnos que realizan un desayuno completo, Calificación relacionada con la capacidad de concentración del estudiante corroborado por el profesorado. Los resultados hallados indican que conforme avanza la calidad del desayuno, el promedio se incrementa, la diferencia se da especialmente en los grupos de desayunó de mala y buena. Las diferencias mayores se hallan en las notas del grupo de alumnos que no desayunan

En general se puede decir que la población de estudiantes consume un desayuno poco adecuado lo que puede afectar a sus resultados académicos, otros estudios establecen que hay una relación estadística directa entre la calidad del desayuno y el rendimiento académico de los alumnos. 


\section{REFERENCIAS BIBLIOGRÁFICAS}

Bayano, López, y Galileo G. (2014). Estudio sobre el desayuno y el rendimiento Académico. Navia-ASTURIAS, 3, 2

Chávez, Santo Domingo, Muradas y Fonos, (1998). Estudio de la ingesta alimentaria de un colectivo homogéneo de Niños de ambos sexos y de edades comprendidas entre seis y quince años. Nutr Clin, 10,26-8.

Farré, Frasquets, Martínez, y Roma, (1999). Dieta habitual de un grupo de adolescentes valencianos. Nutr Hospt; $14,223-230$

Fernández, Aguilar, Mateos, Martínez, (2008). Relación entre la calidad del desayuno $y$ el rendimiento académico en adolescentes de Guadalajara (CastillaLa Mancha)

Herrero, y Fillat, (2008). Estudio sobre el desayuno y el rendimiento escolar en un grupo de adolescentes. Nutr Hosp. 21(3), 346-352

López, De Andraca, Perales, Heres, Castillo, y Colombo, (1997). Breakfast omission and cognitive perfomance of normal, wasted and stunted school children. Eur J Clin Nut.; 47,533-542

Ministerio de Salud Perú (2012). Requerimiento de Energía de la población peruana.

Nicklas, O'Neil, Berenson GS(1998). Nutrient contribution of breakfast, secular trends, and the role of ready-to-eat cereals: a review of data from Bogalusa
Heart Study. Am J Clin Nutr. (suppl),757-763.

Núñez, Cuadrado, Carbajal, y Moreira (1998). Modelo actual de desayuno en grupos de diferente edad: niños, adolescentes y adultos. Nutr Hospt, 13,193-198.

O'Neil y Berenson, Nutrient contribution of breakfast, secular trends, and the role of ready-to-eat cereals: a review of data from Bogalusa Heart Study. Am J Clin Nutr 1998, (suppl),757-763

Pérez, Magra, Rivero, y Santo, (1998). Importancia del desayuno en el rendimiento escolar. (eds.). Barcelona: Ed. Sal vat, p.285.

Preziosi, Galán, Deheeger, Yacoub, y Drewnowski, (1999). Breakfast type, daily nutrient intakes and vitamin and mineral status of French children, adolescents an adults. J Am Coll Nutr, $18,171-178-2008$

Sánchez, y Sierra, (2007). Importancia del desayuno en el rendimiento intelectual $y$ en el estado nutricional de los escolares Universidad de Las Palmas de Gran Canaria. Rev Esp Nutr Comunitaria, 6(2), 53-95

Serra, y Serra, (2004). Desayuno y equilibrio alimentario. Estudio en Kid. Barcelona: Masón.

Correo electrónico:

Revisión de Pares:

Recibido: 02 - 03 - 2018

Aceptado: 15 - $06-2018$ 